\title{
The Effect of TRIZ on Research Institute Innovation
}

\begin{abstract}
With a background in improving independent innovation capabilities and building a world-class petroleum research institute, scientific innovation is the essence and basis of an innovative framework for a research institute, as well as the inexhaustible driving force for sustainable development. By introducing the theory of inventive problem solving (TRIZ) and combining it with CAI (computer aided innovation) technology, the Research Institute of Petroleum Exploration and Development established an adaptive innovative system by making experiments and fanning out from a point to an area gradually. This article takes the example of the innovative practices of the Department of Petroleum Equipment in Research Institute of Petroleum Exploration and Development, and introduces a building method for the innovative system based on technical innovation, enterprise culture, organizational structure, scheme, and people. It also summarizes the experiences and effects of improving innovative system upon scientific practice which can provide important guidance and reference significance for accelerating independent innovative capability.
\end{abstract}

Keywords: TRIZ theory, innovative system, innovative capability, petroleum equipment, research institute

\section{Introduction}

The Research Institute of Petroleum Exploration and Development (RIPED) was founded in 1958. It is a comprehensive research institute under China National Petroleum Corporation (CNPC) for exploring oil and natural gas around the world. Its business involves, among other things: (1) oil-gas exploration; (2) new energy exploration; (3) technical training; and (4) post graduate education. With a background of improving independent innovative capabilities and building a world-class petro-

Manuscript received July 30, 2014; accepted October 30, 2014

He Liu ( $ه$ ), Qing-hai Yang, Xu Jin

The Research Institute of Petroleum Exploration and Development, Beijing 100083, China

Email: liuhe@petrochina.com.cn leum research institute, scientific innovation is the essence and basis of the institute's innovative framework. It is an inexhaustible driving force for sustainable development. Scientific innovation plays an increasingly important role in improving the competitiveness of the research academy, and has drawn more and more attention. In 2009, the RIPED introduced theory of inventive problem solving (TRIZ). How to integrate this highly efficient technical innovation tool into a research academy is an important task before all petroleum professions eager to innovate. The RIPED managed to establish an adaptive innovative system in terms of making experiments and fanning out from a point to an area gradually while constantly improving it in practice, building a good example, and providing a leading role in this regard.

\section{The essence of TRIZ theory}

Theory of Inventive Problem Solving (free translation from Russian Teoriya Resheniya Izobreatatelskikh Zadatch, abbreviated as TRIZ; or as TIPS) is a systematic methodology of problem solving based on knowledge and serving human needs. TRIZ theory was invented by Genrich S. Altshuller in 1946. In the following decades, he focused on the research and improvement of TRIZ theory. As a result of his initiative, dozens of research institutes, universities and enterprises in the Soviet Union established TRIZ research groups and analyzed almost 2.5 million high level patents from around the world. The common rule and deviation model for different technology developments was then summarized. Innovation theory and principles for tackling different kinds of technical problems and physics conflicts were also summarized. A comprehensive theoretical system composed by many methodologies and algorithms in solving technical problems and realizing innovation was established. By combining these principles and rules of multiple disciplines, a theoretical system for TRIZ was established. There are two key points of TRIZ. First, the evolution of all products or technical systems accords with certain rules similar to those of biologic systems, and can be mastered, followed and utilized by people. Second, despite the characteristics and differences 
of innovation for different sectors, the basic technical principles are interlinked. The technical problems in one sector can usually be solved more efficiently by making reference or using principles from other sectors (Fu, 2013).

\section{Applying the TRIZ innovation method}

Methodology is crucial in scientific innovation. To change the innovation mindset, improve independent innovation and research capability, the RIPED combined TRIZ theory and CAI technology organically, and achieved the fast leap in innovation models from "no idea" to "have an idea", and then to "a better idea". The RIPED adopted the model of guidance-training-application, and used TRIZ theory in innovation practice gradually in petroleum science.

\subsection{Guiding by innovative mindset}

The RIPED educated and guided the innovative mindset of administrative personnel and combined it with the company strategy organically (Wang, Wang, \& Sun, 2012). We adopted centralized training and specific counseling to form the overall understanding and extent of importance of the TRIZ theory. The administrative people then realized the significance of the "Program of constantly improving company technical innovative capability," accepted and applied TRIZ in understanding that it can greatly improve the innovative capability and scientific strength of research academy without increasing input.

\subsection{Popularizing by training}

The RIPED popularized TRIZ training, by means of on-site and video conferencing, helping the trainees to accept TRIZ and understand the huge potential value of combining CAI technology and innovation capability. Technical people realize that TRIZ is something like the Master Sun's Art of War to them by accumulating innovation methods. TRIZ can be regarded as a key to unlocking critical problems of industry-university- research cooperation by facilitating the cultivation of innovation mindset sand improvement of innovation capabilities (Wang, Wang, \& Sun, 2012). Through popularizing TRIZ by training, scientific people have gotten the key to innovation, especially source innovation.

\subsection{Improving application capability}

Under the influence of TRIZ theory, engineering thinking has become more clear and logical, and the capability for solving concrete problems substantially increased. Technical discussions of projects under research according to TRIZ are conducted finding some useful solutions. In the
Study on Drilling Rig Power Swivel projects engineers from the Department of Petroleum Equipment realized the foundation of the research and development is Substitute Principle of Mechanical System was to set up a shift gear in the planetary reduction gear to replace torque booster. The unique function of the planetary reduction gear makes top drive device performance much better than traditional ones. By comparative study, the technical route for the innovation process has been clarified. People have gained new cognition and passion to apply TRIZ because they believe it is a pragmatic tool.

\section{Construction of innovation system}

The construction of an innovation system is the fundamental assurance to the improvement of the research and development capability and the core-competitiveness of research academy; and to the realization of sustainable development. The RIPED has built many innovation systems suitable for different specialties in the institute based on individual research areas according to the characteristics of each department.

In the process of learning and practicing, the Department of Petroleum Equipment, which was more suitable for the application of TRIZ was chosen as the pilot unit. It gradually changed the technical innovation activities of the institute to conscious, constant, and organized activities under overall administration. It established an innovation system based on factors of technical innovation strategy, enterprise culture, organizational structure, scheme, and people, and took a demonstration effect.

\subsection{Technical innovation strategy}

Technical innovation is one of the key factors ensuring development of the research academy. The Department of Petroleum Equipment integrates this technical innovation strategy into the development strategy for the institute, and draws up specific short-term, mid-term and long-term technical innovation strategies according to the development status and trend of oil-gas drilling and production equipment. It gradually integrates technical innovation into daily scientific activities as a routine practice and forms an innovation model suitable for the development of oil-gas equipment.

4.1.1 Cultivating and deepening an innovative mindset and innovative ideas

The Department of Petroleum Equipment has clarified the basic innovation idea in terms of years research and development practice of drilling and production equipment, i.e. the essence of innovation is to break the current 
mindset, to absorb useful ideas and to form new idea of development. By doing this, the company can boost its vigor and maintain sustainable development.

In traditional thinking, innovation is regarded as complex boring work with no rules. The first step toward breaking this traditional innovation thinking is the first step to real innovation. In this context, the Department of Petroleum Equipment established an overall plan and objectives for different phases to be implemented. It periodically propagandizes innovation thinking to leaders, project managers, and engineers. It provides systematic training on innovation theory and method to enable them to master key theories and the latest research into technical innovation. It helps to form unique innovation patterns and breaks traditional mindset limits. With a deeper understanding of innovation and its theory and method, scientists and engineers have clearer and more logical mindsets and have systematically mastered a theory and methodology of tackling scientific problems.

\subsubsection{Establishing measures to ensure innovation}

(1) Fully respect the leading role of the scientific personnel. The Department of Petroleum Equipment fully respects the leading role of key scientific personnel in spreading and utilizing innovative methods. By implementing the methods in specific projects, an active atmosphere of learning and applying innovative methods has been established. The overall innovative capability of scientific personnel has been promoted and transferred to the driving force of the development.

(2) Establish innovation team. The Department of Petroleum Equipment established a scientific innovation team and divided it into several small groups according to the practical situation. The innovation group is led by mainstream engineers and composed of members who have participated innovation theory and method training. The innovation groups meet every two weeks to hold technical discussions in order to, among other things: maintain parallel developments for each working item; monitor content which covers problems emerging in the project; submit and display patents; and become aware of new technological breakthroughs.

(3) Strengthen academic exchange in innovation. The Department of Petroleum Equipment has been exploring the forms of academic exchange in terms of such things, as: technical salons; academic seminars; innovation brainstorm meetings; and, thematic workshops. It has normalized study and exchange, and enhanced the involvement, attractiveness and effect of innovation study to build an innovation learning team.

(4) Set up incentive and insurance system. Patent submission and the number of theses published are combined with individual reward, promotion, vacation, and model staff selection so as to encourage "work more, get more" via double incentives to outstanding innovative experts both physically and spiritually.

\subsubsection{Practical application of innovation theory}

(1) Strengthen the inheritance and development of the results of previous studies and form new awareness of innovation theory. In the process of applying innovation theory, researchers collected and regulated the outcomes and patented technologies from former researchers and place them in accord with the innovation theory of TRIZ by analyzing existing innovation outcomes, and then forming an intuitive cognition of the TRIZ innovation principle.

(2) Master innovation skills and expand applications of technical innovations. In the process of project research and development, the steps of application of the innovation methods are mastered. The problems are identified and defined including how to specify the concept to solve the problem. On this basis, the practical application and improvement of the technical innovation in scientific research projects was deepened.

\subsection{Organizational structure}

To effectively implement relevant content into technical innovation strategy, in training, the Department of Petroleum Equipment designed a correspondent organization structure and improved its flexibility and adaptability in order for the innovative organization to enable the new organization to satisfy relevant requirements of technical innovation.

\subsubsection{Vertical structure}

An innovative organization adopts a system of combining unified leadership and hierarchical direction. The Department of Petroleum Equipment has five divisions: a Comprehensive Laboratory; a Bionic Engineering Laboratory; a Technique Completion Laboratory; a Wellbore Control Laboratory; and an Artificial Lift Laboratory. The Comprehensive Laboratory coordinates specialization and cooperation between research units. Other research units set up relatively independent science research innovation teams, which are further divided into several innovation groups according to the research area. Members of the innovation groups are the mainly technical backbone. The innovation group holds both regular and irregular, internal technical discussions and exchange discussions in order to communicate the research and reserve project updates.

\subsubsection{Horizontal structure}

Research units are interrelated and mutually independent. The research unit technical innovation group organizes 
distinctive innovation activities according to its strength and the course of action as well as the method of the project research and development. It also adjusts the course of action and method according in a timely manner for the research progress of the project to form a highly flexible and adaptive horizontal system for job divisions and cooperation. The relationship among different units is independent as well as cooperative and until project target realization is obtained.

\subsection{System}

The research academy wants to develop a system in accordance with its strategy and keep improving it in order to avoid the inconsistency and discordance of the system in deviating the strategy. It promotes technical innovation into the company to assure that technical innovation is conducted on a regular and consistent basis while constantly boosting innovation passion and activity of scientific personnel. It is driving force for innovation in terms of system, scheme, the mental, and the physical to build a superior atmosphere for innovation. In the strategic application process of technical innovation, it actively participates in national key research projects and facilitates independent project approval by the research academy. It also promotes the dual system construction in administrative and technical domain, and improves and implements innovation incentive system in conjunction of innovation plans.

\subsection{Corporate culture}

The Department of Petroleum Equipment integrates technical innovation into corporate culture to make innovation activity active, conscious, and constant. It promotes an objective management approach and builds outstanding culture and an atmosphere of "three simple, three loose". "Three simple" means a simple working environment, simple working procedures, and a simple working style. "Three loose" means a loose academic environment, loose academic demeanor, and loose academic style. By integrating the above three organically, all are encouraged to innovate.

4.4.1 Building a strong innovative atmosphere in terms of both software and hardware

Good tools and experimental methods are provided to the research personnel to boost their creativity, by equipping them with professional instruments, software and software and by forming an outstanding research and development atmosphere in terms of software and hardware. In the meantime, a positive cultural atmosphere is created as the soft environment to encourage combatant spirit for scientific research.
4.4.2 Strengthen humanity building and unify scientific spirit and humanity

The Department of Petroleum Equipment highlights not only instrumental rationality but value rationality as well. It creates an atmosphere of encouraging innovation, respecting talents, academic democracy and tolerating failure. It constantly cultivates an economic mindset of science personnel that participate in market competition and relevant dedication.

\subsection{Human resources}

Human resources are critical to implementing a technical innovation strategy. Talented people are the active body of technical innovation. Team building is one of the fundamental constructs of technical innovation. The Department of Petroleum Equipment set up a science team in accordance with strategic thinking at the same time as organizing and designing. It designated most appropriate work to relevant people and made research and development personnel at all levels to have the mindset and working style in accordance with the strategy of the research academy.

By using the ladder-shaped model to train key technical personnel and strengthen the team building, the research department has cultivated a batch of backbone people with focus, encouraged passing on experience, and sent young staff to the front line of the oil field to learn.

\section{Demonstrated outcomes}

As a pilot institute applying TRIZ and CAI, the Department of Petroleum Equipment has collected and analyzed techniques and scientific outcome for oil extraction equipment, drilling machines, and down hole tools. It has developed a unique innovation theory system for Department of Petroleum Equipment. It has accelerated an innovative application of TRIZ theory and CAI technique in the research and development of oil and gas drilling and production equipment.

5.1 The practice of the application of TRIZ theory in the research and development of expansion pipe technique

Through practice, the technique of expansion pipe has got innovative breakthrough that addressed key technical issues such as tailpipe suspension, sidetrack drilling and large supply pipe and developed the technique of open hole expansion pipe completion and relevant tools, as shown in Figure 1. The technique has been used in more than 100 wells in 11 oil and gas fields in failed well repairing and conforming control. It has been awarded more than 20 national patents with 5 for invention. 


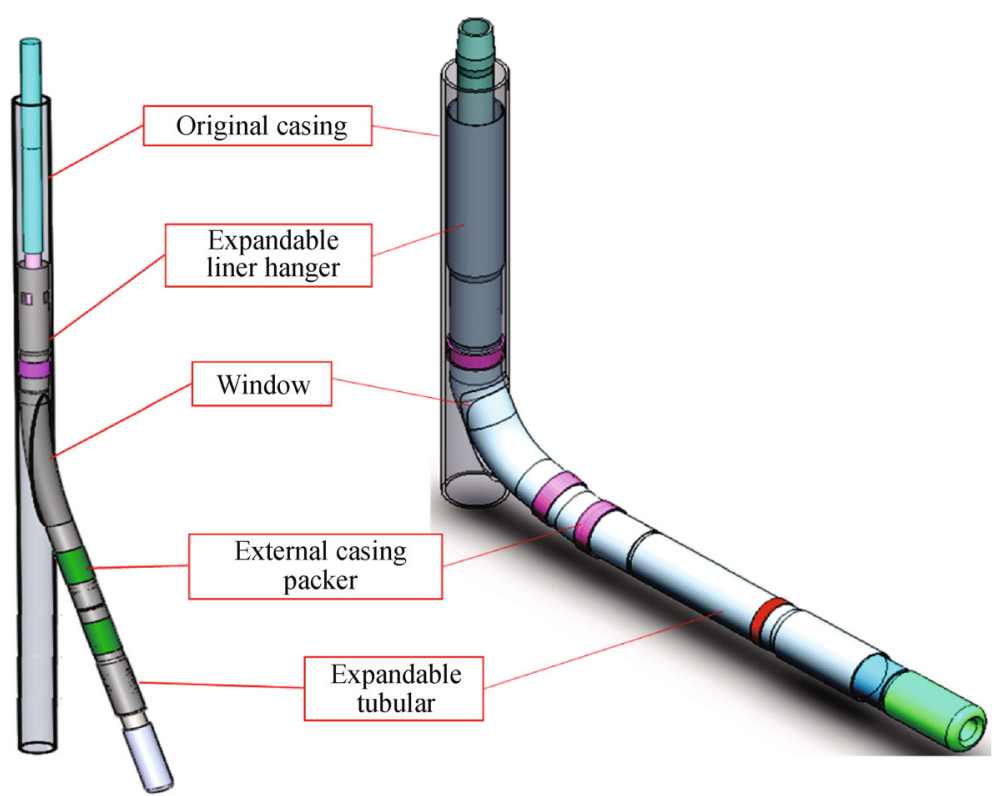

Figure 1. Well completion tools model diagram of sidetracking well expandable tubular.

5.2 The research on TRIZ applications in oil field well completion

By applying TRIZ to the design and optimization of well completion tools, remarkable results have been achieved. Innovative design of a number of advanced tools such as Interlayer Control Valve, Self-expandable Packer, Multiflow Control Valve have been completed. 10 national patents have been obtained with 5 for invention. It was applied on site in more than 30 wells and formed 3 new techniques for Open Hole Selective Completion and Production Control Technology in Horizontal Well, Sand Control, and Inflow Control Device (ICD) Technology and 11 kinds, and 32 sets of new well completion tools.

\section{Conclusions}

Practice shows that in solving problems in different sectors have common rules. TRIZ innovation theory contains general innovation methods and principles which can be widely in many technical areas. The successful combination of TRIZ and CAI technology highlighted the importance of innovation theory. The successful application of the theory by the Department of Petroleum Equipment of research academy enhanced its cognition, and served as a good model with facilitating effect. It has an obvious applications to scientific innovation in oil and gas equipment which has significant reference in expanding innovation channel and promoting innovative thinking. In the meantime, the innovation outcome of the research and development of oil and gas equipment greatly contributed to improving the independent innovative capabilities of the research institute, which further provides more important technical support for the sustainable development of China's oil industry.

\section{References}

Fu, Y. (2013). The discussion of promoting TRIZ innovation theory in companies. Gansu Sci-Tech Prospects, 42, 8-9

Wang, X., Wang, H., \& Sun, X. (2012). The management research of optimizing regional innovation platform based on TRIZ. Science and Technology Progress and Actions, 15, 1-6 